\title{
Power law of decaying homogeneous isotropic turbulence at low Reynolds number
}

\author{
P. Burattini, ${ }^{1, *}$ P. Lavoie, ${ }^{2}$ A. Agrawal,${ }^{3}$ L. Djenidi, ${ }^{2}$ and R. A. Antonia ${ }^{2}$ \\ ${ }^{1}$ Physique Statistique et Plasmas, Universite Libre de Bruxelles, B-1050 Brussels, Belgium \\ ${ }^{2}$ Department of Mechanical Engineering, University of Newcastle, 2308 NSW, Australia \\ ${ }^{3}$ Department of Mechanical Engineering, Indian Institute of Technology, Powai, Mumbai 400076, India
}

(Received 24 November 2005; published 8 June 2006)

\begin{abstract}
We focus on an estimate of the decay exponent $(\mathrm{m})$ in the initial period of decay of homogeneous isotropic turbulence at low Taylor microscale Reynolds number $R_{\lambda}(\simeq 20-50)$. Lattice Boltzmann simulations in a periodic box of $256^{3}$ points are performed and compared with measurements in grid turbulence at similar $R_{\lambda}$. Good agreement is found between measured and calculated energy spectra. The exponent $m$ is estimated in three different ways: from the decay of the turbulent kinetic energy, the decay of the mean energy dissipation rate, and the rate of growth of the Taylor microscale. Although all estimates are close, as prescribed by theory, that from the Taylor microscale has the largest variability. It is then suggested that the virtual origin for the decay rate be determined from the Taylor microscale, but the actual value of $m$ be estimated from the decay rate of the kinetic energy. The dependence of $m$ on $R_{\lambda}(0)$ (the value of $R_{\lambda}$ at the beginning of the simulation) is also analyzed, using the present data as well as data from the literature. The results confirmed that $m$ approaches 1 , as $R_{\lambda}(0)$ increases.
\end{abstract}

DOI: 10.1103/PhysRevE.73.066304

PACS number(s): 47.27.Gs, 47.27.T-

\section{INTRODUCTION}

Homogeneous isotropic turbulence (HIT) has long been at the core of turbulence research. Since the seminal work of Rogallo [1], direct numerical simulations of HIT have helped to clarify, inter alia, the scaling properties of turbulence, the effect of the Reynolds number $\left(R_{\lambda}\right)$, and the behavior of the velocity derivative skewness. At present, the highest- $R_{\lambda}$ direct numerical simulations (DNS's) are performed in periodic boxes for stationary conditions, achieved through a continuous injection of energy into the largest scales. DNS's have also been performed for temporally decaying homogeneous isotropic turbulence (DHIT), where the turbulence decreases with time from an initial velocity distribution, in the absence of kinetic energy production. It has been recently suggested that some basic properties of turbulence may differ between stationary and decaying conditions, even at the same $R_{\lambda}$ [2,3]. Further, it has been noticed [4,5] that, in pursuing ever higher values of $R_{\lambda}$ and focusing on the smallest scales, proper resolution of the largest scales may have been overlooked. DHIT, although limited in the initial period of the decay (where a power law is established) to lower values of $R_{\lambda}$ compared to stationary HIT, is potentially less affected by this problem. DHIT is appealing because, compared to shear flows, it avoids the complications related to a source of turbulent energy, while the properties of homogeneity and isotropy reduce sensibly the mathematical complexity of the analysis.

The experimental analog of DHIT is grid turbulencee.g., $[6,7]$. In this case, the turbulence intensity decreases in the streamwise direction, as the fluid moves with a constant mean velocity away from the grid. In a frame of reference moving with the mean velocity, turbulence decays in time and is approximately homogeneous.

*FAX: +32 (0)2 650 5824. Electronic address: Paolo.Burattini@ulb.ac.be
HIT has been typically studied with the computer using finite-difference (e.g., [8]) or spectral methods (e.g., [1,9]). In this paper, we investigate DHIT by solving the lattice Boltzmann equation and we compare the results-including the kinetic energy spectrum and the velocity derivative skewness-to measurements we made in grid turbulence. The values of $R_{\lambda}$ and the Mach number are relatively low. In the literature, there are a few simulations of HIT done with the lattice Boltzmann method (LBM) [10-12]. This method offers advantages in terms of parallelization of the algorithm for high-performance computing (e.g., [11]) and avoids the need of solving Poisson's equation for the pressure.

The main focus, here, is on temporal profiles of turbulent kinetic energy, its mean dissipation rate, and the Taylor microscale. Many studies have investigated the decay laws and similarity in DHIT [6,13-20] because these two properties allow useful generalizations in turbulence theory. In particular, Huang and Leonard [20] studied similarity and the power-law decay of box turbulence at low Reynolds number, $5<R_{\lambda}<50$, using spectral DNS's. They found that the exponent $m$ for the kinetic energy decay $q^{2} \sim t^{-m}$ ( $t$ is time) falls in the range $1.25<m<1.5$. They also reported on the dependence of $m$ on $R_{\lambda}$. In agreement with George [18], they showed that $m \rightarrow 1$ for increasing $R_{\lambda}$. In this work, we propose a refined procedure to obtain $m$ in a reliable way. The relationship between $m$ and $R_{\lambda}$ is of importance-for instance, in modeling small-scale turbulence. A recent work has addressed grid turbulence and its decay laws using LBM simulations [21].

\section{NUMERICAL DETAILS AND INITIAL CONDITIONS}

DHIT is simulated in a periodic box of $256^{3}$ points. The Boltzmann equation is solved on a lattice for the discrete velocity distribution

$$
f_{\ell}\left(\mathbf{X}+\delta_{x} \mathbf{e}_{\ell}, t+\delta_{t}\right)-f_{\ell}(\mathbf{X}, t)=\Omega_{\ell}, \quad \ell=0, M,
$$

where $f_{\ell}$ is the particle distribution function, $\mathbf{e}_{\ell}$ is the velocity along the $\ell$ th direction, $M$ is the population size, $\Omega_{\ell}$ is the 
TABLE I. Main parameters for the LBM simulations of box turbulence.

\begin{tabular}{llllllllllll}
\hline \hline Case & $\omega$ & $k_{p}$ & $\sigma$ & $t_{\max }$ & $R_{\lambda}(0)$ & $\overline{m_{q}}$ & $\overline{m_{\lambda}}$ & $\overline{m_{\epsilon}}$ & $L(0) k_{\min }$ & $\eta k_{1 \max }$ & $t_{0}$ \\
\hline $\mathcal{A}$ & 1.984 & 9 & 4 & 9.7 & 170 & 1.39 & 1.34 & 1.39 & 0.33 & 1.0 & 1.08 \\
$\mathcal{B}$ & 1.98 & 3 & 4 & 11.9 & 582 & 1.19 & 1.14 & 1.02 & 0.84 & 1.2 & 4.3 \\
\hline \hline
\end{tabular}

collision operator, $\mathbf{X}$ is the position vector, $t$ is time, and $\delta_{x}$ and $\delta_{t}$ are the lattice space and time steps. The single relaxation time approximation [Bhatnagar-Gross-Krook (BGK) model [22]] for the collision operator has been used-i.e.,

$$
\Omega_{\ell}=-\omega\left[f_{\ell}(\mathbf{X}, t)-f_{\ell}^{e q}(\mathbf{X}, t)\right],
$$

where $f_{\ell}^{e q}$ is the local equilibrium distribution and $\omega^{-1}$ is the relaxation time, related to the viscosity. The lattice has unit lengths in time $\left(\delta_{t} \equiv 1\right)$ and space $\left(\delta_{x} \equiv 1\right)$, also denoted as lattice units (LU's). The mass density and momentum density are calculated as follows:

$$
\begin{gathered}
\rho=\sum_{\ell} f_{\ell}(\mathbf{X}, t), \\
\rho \mathbf{u}=\sum_{\ell} f_{\ell}(\mathbf{X}, t) \mathbf{e}_{\ell},
\end{gathered}
$$

where $\mathbf{u}=\left(u_{1}, u_{2}, u_{3}\right)$. The kinematic viscosity is

$$
\nu=\left(\frac{2}{\omega}-1\right) \frac{1}{6}
$$

and the initial density is set to 0.3 (hereafter, dimensional quantities are expressed in LU's). In this work, a cubic lattice with 15 links $(d 3 q 15[23,24])$, including 14 velocity vectors and a rest state, is used. The speed of sound for $d 3 q 15$ is $c_{s}=\sqrt{3 / 8}$.

The mean energy dissipation rate of the macroscopic velocity fluctuation, $u_{i}$, is defined as

$$
\epsilon=\frac{\nu}{2}\left\langle\left(\frac{\partial u_{i}}{\partial x_{j}}+\frac{\partial u_{j}}{\partial x_{i}}\right)^{2}\right\rangle
$$

(hereafter, unless otherwise stated, repeated indices imply summation; angular brackets denote space averaging in simulations and time averaging in experiments). Assuming isotropy, Eq. (1) reduces to

$$
\epsilon=15 \nu\left\langle\left(\frac{\partial u_{i}}{\partial x_{i}}\right)^{2}\right\rangle
$$

(no summation here). The Taylor microscale and Taylormicroscale Reynolds number are

$$
\begin{gathered}
\lambda=\frac{\left\langle u_{1}^{2}\right\rangle^{1 / 2}}{\left\langle\left(\partial u_{1} / \partial x_{1}\right)^{2}\right\rangle^{1 / 2}}, \\
R_{\lambda}=\frac{\left\langle u_{1}^{2}\right\rangle^{1 / 2} \lambda}{\nu},
\end{gathered}
$$

while the Kolmogorov scale is $\eta=\nu^{3 / 4} \epsilon^{-1 / 4}$. Note that both $\lambda$ and $R_{\lambda}$ are defined using only the component $u_{1}$. The integral length scale is

$$
L(t)=\frac{3 \pi}{4} \frac{\int_{0}^{\infty} k^{-1} E(k, t) d k}{\int_{0}^{\infty} E(k, t) d k}
$$

where $E(k, t)$ is the three-dimensional (3D) energy spectrum.

The initial velocity field is prescribed by assuming the following initial (i.e., $t=0$ ) 3D energy spectrum:

$$
E(k, 0)=\frac{q^{2}(0)}{2} \frac{1}{A} \frac{k^{\sigma}}{k_{p}^{\sigma+1}} \exp \left[-\frac{\sigma}{2}\left(\frac{k}{k_{p}}\right)^{2}\right],
$$

where

$$
q^{2}(t)=\left\langle u_{i}^{2}\right\rangle=2 \int_{0}^{\infty} E(k, t) d k
$$

is twice the mean turbulent kinetic energy $[8,9]$. The value of $q^{2}$ is such that the Mach number is $\ll 1-$ i.e.,

$$
M=\frac{q}{c_{s}} \simeq 0.03
$$

By using the condition (4), the constant $A$ is

$$
A=\int_{0}^{\infty} \frac{k^{\sigma}}{k_{p}^{\sigma+1}} \exp \left[-\frac{\sigma}{2}\left(\frac{k}{k_{p}}\right)^{2}\right] d k=\int_{0}^{\infty} \tilde{k}^{\sigma} \exp \left[-\frac{\sigma}{2} \widetilde{k}^{2}\right] d \tilde{k},
$$

where $\tilde{k}=k / k_{p}$. The parameters $\sigma$ and $k_{p}$ fix the initial conditions: the value of $k_{p}$ controls the location of the peak in the initial energy spectrum, while $\sigma$ determines the slope of the spectrum for $k \rightarrow 0$. The lower $k_{p}$ is, the higher the initial value of $R_{\lambda}$-and consequently the higher $R_{\lambda}$ is during the decay. de Bruyn Kops and Riley [4] showed that if $k_{p}$ is too small, such that $L k_{\min }<0.3$, the energy is removed too quickly from the large scales. This may compromise the initial stage of the decay $[5,20]$. The maximum and minimum wave numbers resolved in the present simulations are $k_{1 \max }=2 \pi / 2 \delta_{x}=\pi$ and $k_{1 \min }=2 \pi / N$. The phase of the spectral components is initialized by random numbers (see [25]). This produces an initial velocity distribution which is Gaussian (e.g., the velocity derivative skewness is zero).

In the present study, we simulate two sets of initial conditions (see Table I): case $\mathcal{A}$, for $k_{p}=9$ and $\sigma=4$ (which is the same slope of the von Kármán spectrum-e.g., [26]) and case $\mathcal{B}$, for $k_{p}=3$ and $\sigma=4$. The latter achieves a larger $R_{\lambda}$, comparable to values in our experiment. The simulations of case $\mathcal{B}$ are comparable to those of Antonia and Orlandi [8] and Mansour and Wray [9], who used the same $\sigma$ and $k_{p}$, while studying decaying box turbulence at similar resolutions $\left(240^{3}\right.$ and $270^{3}$ for [8] and $256^{3}$ for [9]). Hereafter, length and velocity scales are made dimensionless with $N$ 
and $\left[q^{2}(0) / 2\right]^{1 / 2}$, respectively, while time scales are normalized by $N /\left(q^{2}(0) / 2\right)^{1 / 2}$, as in $[8,9]$.

To obtain the initial velocity field in physical space, the inverse Fourier transform is applied to the energy spectrum (3). Although the resulting provisional field is, by construction, divergence free in wave-number space, it is not in physical space. An iterative solution of Poisson's equation results in a solenoidal velocity field (see [25]), which can be used to initialize the distribution function $f_{\ell}$ for the LBM simulations. This procedure is different from that proposed by [10], where the initial distribution functions are calculated iteratively from the inverse-transformed velocity components, but without solving Poisson's equation. If neither of the two procedures is followed, strong pressure fluctuations develop at the beginning of each simulation, eventually compromising the entire solution. A discussion regarding the initial pressure field in lattice Boltzmann simulations (of 2D turbulence) can be found in [27].

The code for the simulation has been parallelized with MPICH2 to run on a Beowulf cluster of 16 dual-CPU PC's.

\section{EXPERIMENTAL DETAILS}

A low-speed wind tunnel, with a working section of $350 \times 350 \mathrm{~mm}^{2}$ and $2.4 \mathrm{~m}$ long, is used. Grids and a honeycomb section are located in the plenum chamber to homogenize and straighten the flow. A biplane square mesh grid, made up of $4.76 \times 4.76 \mathrm{~mm}^{2}$ square rods of solidity 0.35 and mesh size $M=24.8 \mathrm{~mm}$, is placed downstream of the primary contraction (having an area ratio of 9:1) at the beginning of the working section (which is the origin of the $x$ axis in the streamwise direction). A secondary, axisymmetric contraction (area ratio 1.36) is placed after the grid, in order to improve the isotropy of the flow. The ratio $\left\langle u_{1}^{2}\right\rangle /\left\langle u_{2}^{2}\right\rangle$ is about 1.05 , instead of 1.17 without the contraction [28] $\left(u_{1}\right.$ is the component along the streamwise direction). More details on the experimental setup can be found in [29]. Measurements are made on the centerline of the working section at $x=40 M$ from the grid, where the turbulence is nearly homogeneous and isotropic, according to the criteria outlined in [17]. The mean freestream velocity $U$ is about $6 \mathrm{~ms}^{-1}$.

The velocity calibration is performed in situ, after removing the grid. The reference velocity is measured with a Pitotstatic probe connected to a differential pressure transducer (Furness $\mathrm{FCO} 15$, least count $0.024 \mathrm{~mm}_{2} \mathrm{O}$, full range $10 \mathrm{~mm} \mathrm{H}_{2} \mathrm{O}$ ). The streamwise velocity is measured with a single hot wire, operated with in-house constant temperature anemometer (CTA) circuits. The hot wire is etched from Pt$10 \% \mathrm{Rh}$ to a diameter of $d_{w}=2.5 \mu \mathrm{m}$, and the active length $l_{w}$ is chosen so as to have an aspect ratio $l_{w} / d_{w}$ of nearly 200. The CTA circuits are operated at an overheat ratio of 1.5, with a cut off frequency of approximately $15 \mathrm{kHz}$. The anemometer signals are acquired by means of a 16-bit AD board into a $\mathrm{PC}$ at a sampling rate of $f_{s}=8 \mathrm{kHz}$, with lowpass analog filters at $f_{s} / 2$. Taylor's hypothesis is used to convert temporal variations into spatial variations-i.e., $r_{1}=U / t$ - and the wave number is defined as $k_{1}=2 \pi / r_{1}$.

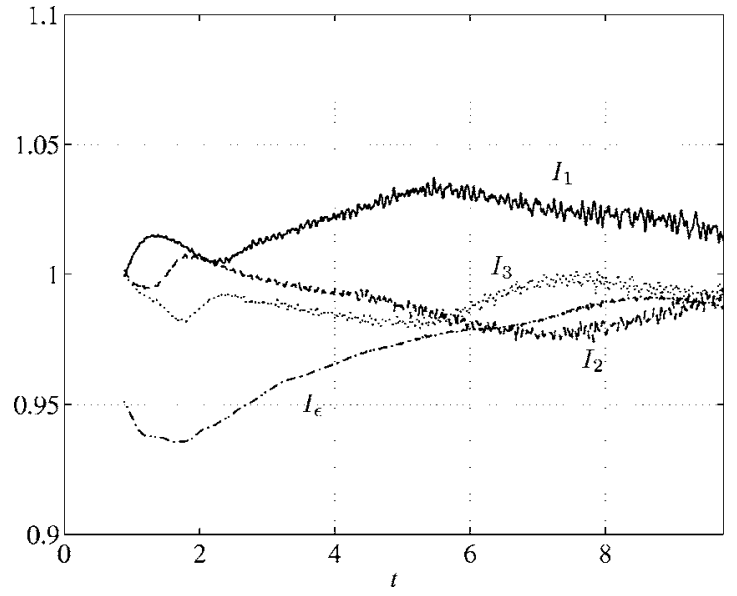

FIG. 1. Isotropy ratios for small and large scales, case $\mathcal{A}$. Solid line: $I_{1}=3\left\langle u_{1}^{2}\right\rangle / q^{2}$. Dashed line: $I_{2}=3\left\langle u_{2}^{2}\right\rangle / q^{2}$. Dotted line: $I_{3}=3\left\langle u_{3}^{2}\right\rangle / q^{2}$. Dot-dashed line: $I_{\epsilon}=\left(\epsilon_{1}+\epsilon_{2}+\epsilon_{3}\right) / 3 \epsilon$.

\section{RESULTS}

\section{A. Basic quantities}

Isotropy at the large scales, quantified by $I_{1}=3\left\langle u_{1}^{2}\right\rangle / q^{2}$, $I_{2}=3\left\langle u_{2}^{2}\right\rangle / q^{2}$, and $I_{3}=3\left\langle u_{3}^{2}\right\rangle / q^{2}$, is shown in Fig. 1 , for $\mathcal{A}$. The values are within $\pm 3 \%$ from 1 , the perfectly isotropic case. The oscillations are not systematic, and indeed they change from one simulation to another, because the initial spectrum contains a random component for the phase. The level of large-scale isotropy achieved here is superior to that in grid turbulence experiments without a secondary contraction [30-32], where $I_{1}$ can exceed 1.2.

Figure 1 shows also the small-scale isotropy quantified by $I_{\epsilon}=\left(\epsilon_{1}+\epsilon_{2}+\epsilon_{3}\right) / 3 \epsilon$, where the numerical subscript identifies which velocity component has been used in evaluating Eq. (2). $I_{\epsilon}$ is within $\pm 2 \%$ from 1 , for $t \gtrsim 4$. Therefore, both large and small scales are isotropic, to a close approximation.

Figure 2 shows the temporal evolution of $\lambda^{2}$ for case $\mathcal{A}$.

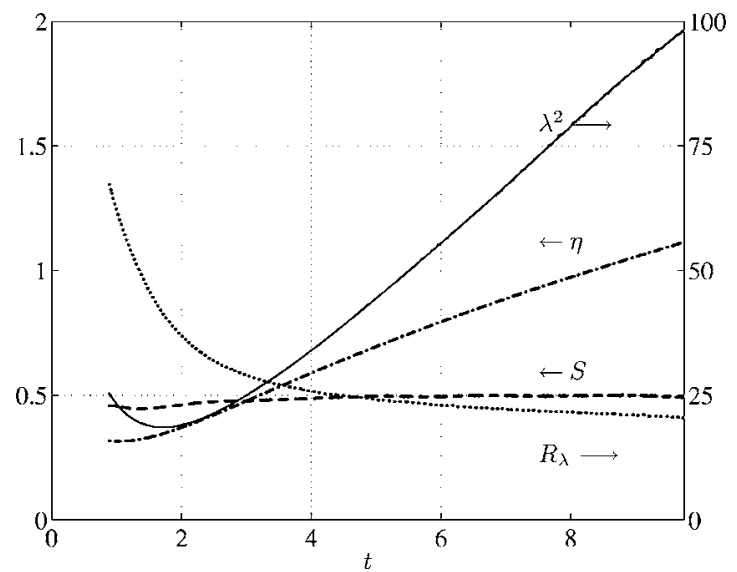

FIG. 2. Profiles of several quantities during the decay, case $\mathcal{A}$. Dotted line: $R_{\lambda}$. Dashed line: velocity derivative skewness. Solid line: Taylor microscale. Dot-dashed line: Kolmogorov scale. A polynomial of order 9 (almost indistinguishable from the data) is fitted to the values of $\lambda^{2}$. 


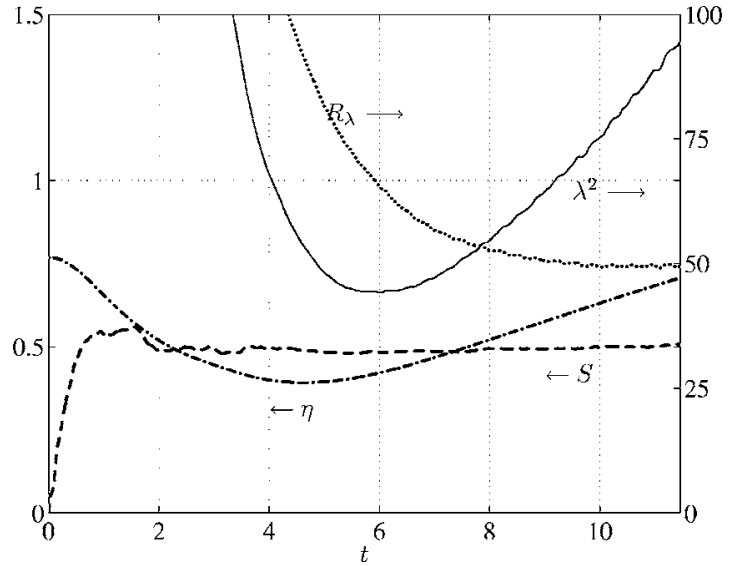

FIG. 3. Profiles of several quantities during the decay, case $\mathcal{B}$. Dotted line: $R_{\lambda}$. Dashed line: velocity derivative skewness. Solid line: Taylor microscale. Dot-dashed line: Kolmogorov scale.

The initial period of decay is identified with the region where $\lambda^{2}$ grows linearly-i.e., for $t \gtrsim 4$. The Kolmogorov scale $\eta$ (same figure) increases during the initial period of decay, after having reached a minimum value of 0.3 at $t \simeq 2$.

The skewness of the velocity derivative,

$$
S_{i} \equiv-\frac{\left\langle\left(\partial u_{i} / \partial x_{i}\right)^{3}\right\rangle}{\left\langle\left(\partial u_{i} / \partial x_{i}\right)^{2}\right\rangle^{3 / 2}}
$$

(no summation implied here), has a value typically close to 0.5 in HIT and approaches zero, as $R_{\lambda} \rightarrow 0$. This has been shown experimentally by Tavoularis et al. [33], and numerically by Mansour and Wray [9] and Herring and Kerr [34]. $S=\left(S_{1}+S_{2}+S_{3}\right) / 3$ (see Fig. 2) is close to 0.5 , for $t \gtrsim 4$, in agreement with numerical [8,9] and experimental [35] data at comparable $R_{\lambda}$. The region where $S$ becomes nearly constant corresponds closely to where $\lambda^{2}$ displays a linear growthi.e., the initial period of decay. The development of $R_{\lambda}$ during the simulation is also reported in Fig. 2: following an initial rapid decay, $R_{\lambda}$ decreases more slowly for $t \gtrsim 4$.

Figure 3 reports the profiles of $\lambda^{2}, \eta, S$, and $R_{\lambda}$ for case $\mathcal{B}$. Although the values of $R_{\lambda}$ are larger compared to case $\mathcal{A}$, the same qualitative observations still apply.

In DHIT, there is no turbulence production so that the turbulent kinetic energy equation reduces to

$$
\epsilon=-\frac{1}{2} \frac{d}{d t} q^{2}
$$

Figure 4 shows $\epsilon$ calculated from its definition [Eq. (1)] and from $d q^{2} / d t$, Eq. (5). The agreement indicates that the present simulation resolves adequately both large and small scales.

Finally, the values of the normalized energy dissipation rate

$$
C_{\epsilon}=\frac{\epsilon L}{\left\langle u_{1}^{2}\right\rangle^{3 / 2}}
$$

are shown in Fig. 5. $C_{\epsilon}$ has been reported extensively in the literature, for both decaying and forced (i.e., stationary) box turbulence. It is widely accepted that $C_{\epsilon}$ achieves a constant

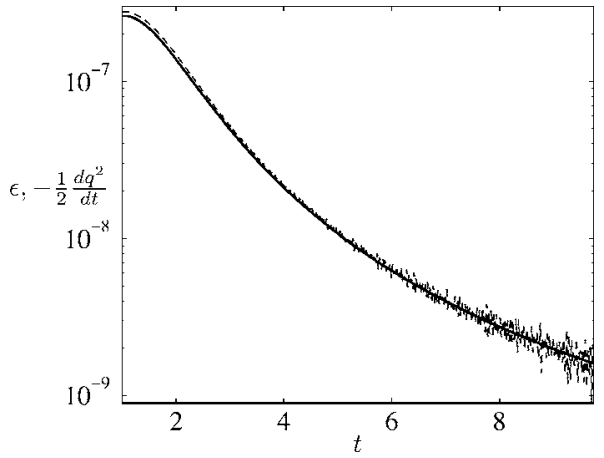

FIG. 4. Profile of the mean energy dissipation rate during the decay, case $\mathcal{A}$. Solid line: from the definition Eq. (1). Dashed line: estimated from $q^{2}$ via Eq. (5).

value near 0.5 , for large $R_{\lambda},(\gtrsim 100)$ [36-38], implying that $\epsilon$ becomes independent of the viscosity, a fundamental assumption of Kolmogorov's theory $[39,40]$. In our simulations, $R_{\lambda}$ is quite small so that $C_{\epsilon}$ is larger than 0.5: for $R_{\lambda} \simeq 24, C_{\epsilon} \simeq 1.26$ and, for $R_{\lambda} \simeq 44, C_{\epsilon} \simeq 0.71$. These values follow the same trend of previously published data (see Fig. 5 which includes data from [41]).

It is interesting to compare simulations and measurements in grid turbulence at similar values of $R_{\lambda}$. The numerical data refer to case $\mathcal{B}$, which achieves larger values of $R_{\lambda}$, close to the experimental ones. The 1D energy spectrum of $u_{1}$, $E_{11}\left(k_{1}\right)$, is given in Fig. 6-after normalizing $k_{1}$ by $\eta$ and multiplying $E_{11}\left(k_{1}\right)$ by $k_{1}^{5 / 3}$. This representation would highlight the presence of the inertial range. However, the small value of $R_{\lambda}(\simeq 49)$ makes such range almost nonexistent. The exponential region of the spectrum, for $k_{1} \eta \gtrsim 0.2$, corresponds to the dissipative range (e.g., [42]). Numerical and experimental distributions are remarkably similar. The agreement is particularly significant because no special care has been taken here, as compared to [4], in matching the initial spectrum in the simulation to that of the experiments, and also because the criterion for the large-scale resolution [4] is

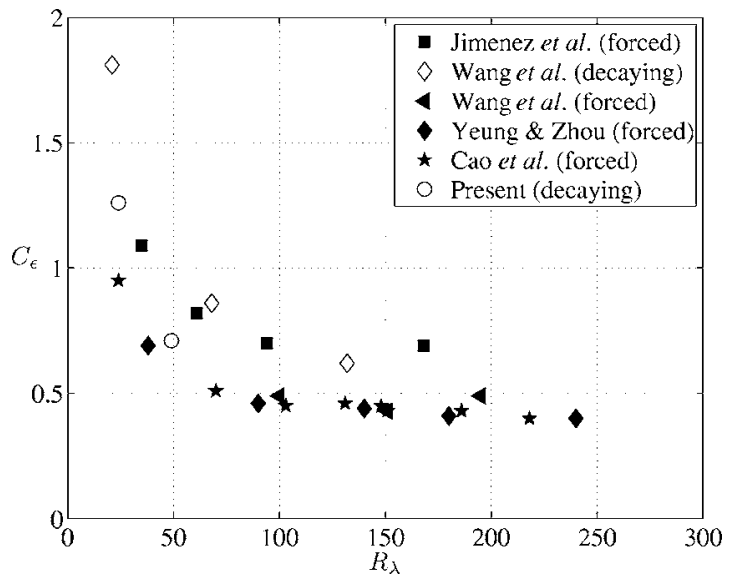

FIG. 5. Distribution of $C_{\epsilon}$ as a function of $R_{\lambda}$ for different numerical simulations of decaying and forced homogeneous isotropic turbulence (adapted from [41]). (ם) Jimenez et al. [45], (४, $\diamond)$ Wang et al. [46], ( ) Yeung and Zhou [47], ( $\star$ ) Cao et al. [48], and $(\bigcirc)$ present. 


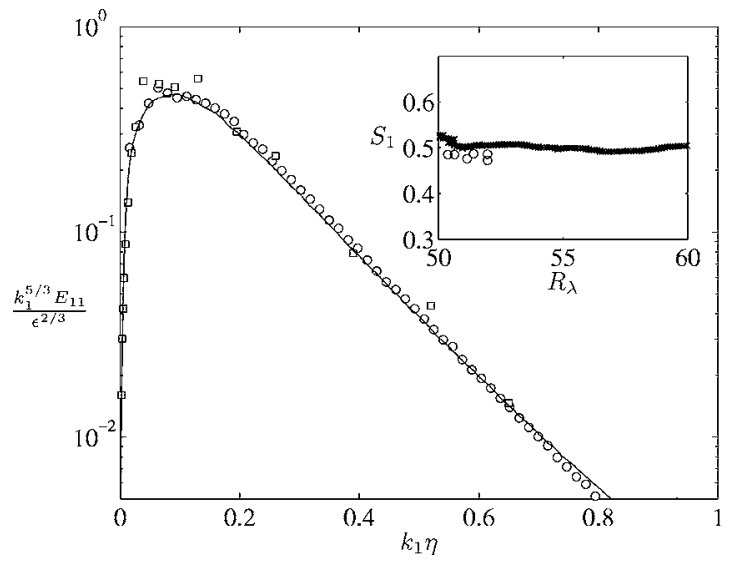

FIG. 6. Spectrum of $u_{1}$ normalized by Kolmorogov scales and compensated by $\left(k_{1} \eta\right)^{5 / 3}$. Solid line: grid turbulence, $R_{\lambda}=49$, present experiment (the original spectrum has been smoothed using a moving average of 20 points). $(\bigcirc)$ box turbulence (case $\mathcal{B}$, $t=10.4$ ), $R_{\lambda}=49$; $(\square)$ grid turbulence, $R_{\lambda}=48.6$, from [43]. Inset: velocity derivative skewness: $(\times)$ box turbulence, $(\bigcirc)$ grid turbulence.

not strictly verified. The grid turbulence data of ComteBellot and Corrsin [43] at $R_{\lambda} \simeq 49$ are also included in the same figure, providing further support for the present results. The inset in Fig. 6 shows the profile of $S_{1}$ : experimental and numerical values are again very close, at comparable $R_{\lambda}$.

\section{B. Power-law decays}

The decay law of the kinetic energy in DHIT has been studied extensively. Its importance is related, among other things, to closure schemes, such as the $k-\epsilon$ model $[44,9]$. It is commonly accepted that, in DHIT, the turbulent kinetic energy decays as a power law-i.e.,

$$
q^{2}=A\left(t-t_{0}\right)^{-m},
$$

where $A$ is the decay constant, $t_{0}$ the virtual origin, and $m$ the decay exponent. These three parameters are usually assumed to be constant along the decay. Batchelor and Townsend [14], who studied decaying grid turbulence, reported a value of 1 for $m$. Subsequently, values consistently larger than 1 have been found. At present, the range $1.15 \leqslant m \leqslant 1.45$ is deemed plausible (e.g., [26]). Equation (5) can be combined with Eq. (6) to yield

$$
\epsilon=\frac{1}{2} A m\left(t-t_{0}\right)^{-m-1} .
$$

From the definition of $\lambda$, it follows that

$$
\lambda^{2}=\frac{15 \nu\left\langle u_{1}^{2}\right\rangle}{\epsilon}=\frac{10 \nu}{m}\left(t-t_{0}\right),
$$

which is linear in $\left(t-t_{0}\right)$. Therefore, differentiating $\lambda^{2}$ with respect to $t$ eliminates the dependence on $t_{0}$ and yields

$$
m=10 \nu /\left(d \lambda^{2} / d t\right) \text {. }
$$

The exponent $m$ can thus be estimated via Eq. (6), (7), or (9), and all three should give the same result, provided Eq. (6) is

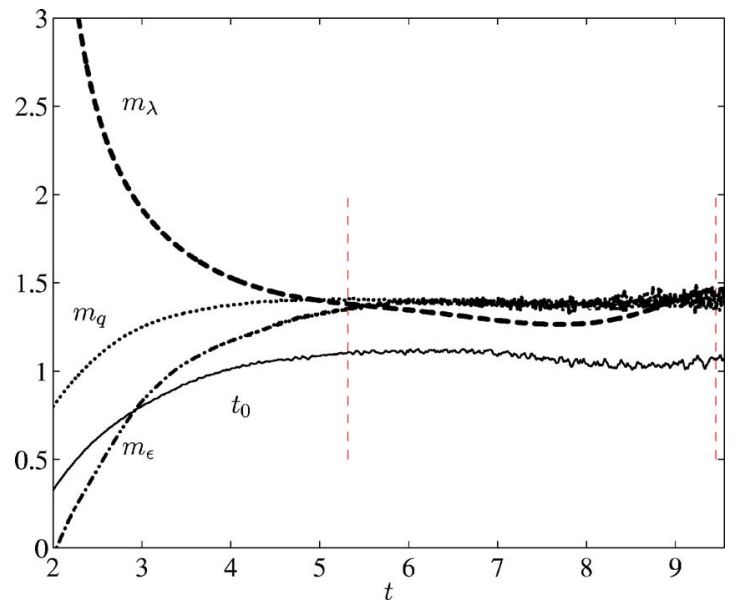

FIG. 7. (Color online) Decay exponents estimated from different quantities (case $\mathcal{A}$ : see Table I. Dashed line: $m_{\lambda}$. Dotted line: $m_{q}$. Dot-dashed line: $m_{\epsilon}$. Solid line: $t_{0}$. Vertical dashed lines indicate the time interval over which the averaged values of $t_{0}$ and $m$ are calculated.

valid. Nonetheless, Eq. (9) has the advantage of not containing $t_{0}$, which can be estimated afterwards from

$$
t_{0}=t-\frac{m}{10 \nu} \lambda^{2}
$$

once $m$ is known. [Conversely, without assuming Eq. (6), if the velocity structure functions of order 2 and 3 satisfy similarity when normalized by $\lambda$ and $q^{2}$, it can be shown that $q^{2}$ decays as a power law [19].]

To investigate possible differences in the estimates of $m$ from Eq. (6), (7), or (9), we now use different subscripts to distinguish between the different quantities on which these estimates are based-viz.,

$$
\begin{aligned}
& q^{2}=A\left(t-t_{0}\right)^{-m_{q},} \\
& \epsilon=B\left(t-t_{0}\right)^{-m_{\epsilon}-1}, \\
& \lambda^{2}=\frac{10 \nu}{m_{\lambda}}\left(t-t_{0}\right) .
\end{aligned}
$$

Here, $B$ is the decay constant for $\epsilon$. The instantaneous values of $m$ are shown as a function of $t$ in Fig. 7. [A polynomial of order 9, almost indistinguishable from the original points (see Fig. 2), has been fitted to the values of $\lambda^{2}$ before taking the derivative.] From the instantaneous values, means (denoted by an overbar) are calculated, by averaging over a time interval (marked by vertical dashed lines in Figs. 7 and 8) where the distributions are nearly constant. Figure 7 shows that, for case $\mathcal{A}, m_{\lambda}$ is almost constant $\left(\overline{m_{\lambda}} \simeq 1.47\right)$ for $5.5 \lesssim t \lesssim 9.5$. In the same interval, $t_{0}$ estimated from $\overline{m_{\lambda}}$ via Eq. (10) is also nearly constant $\left(\overline{t_{0}} \simeq 1.08\right)$. The oscillations in $t_{0}$ are due to the deviation from linearity of $\lambda^{2}$. With $\overline{t_{0}}$ determined, the instantaneous decay exponents from the kinetic energy, $m_{q}$, and from the dissipation, $m_{\epsilon}$, can be estimated via Eqs. (6) and (7), Fig. 7. Although $\overline{m_{\epsilon}}, \overline{m_{q}}$, and $\overline{m_{\lambda}}$ are nearly equal (see Table I), $m_{\lambda}$ displays larger oscillations, 


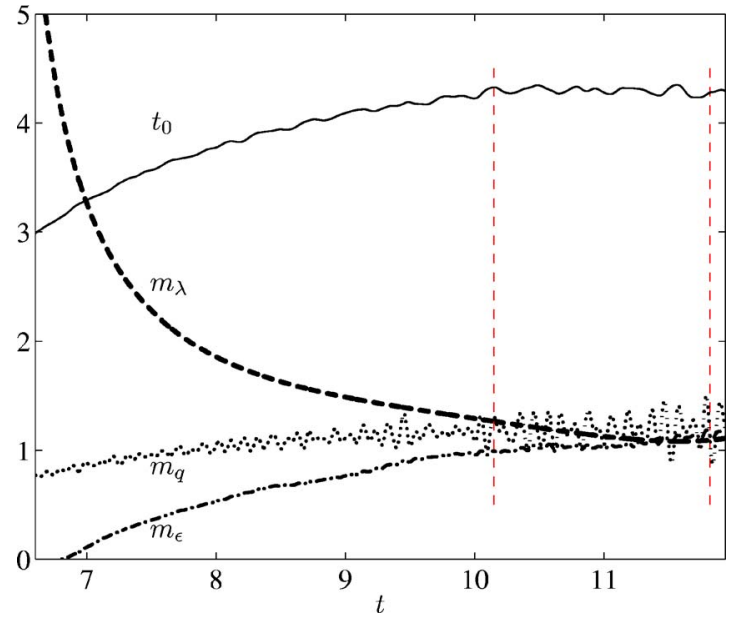

FIG. 8. (Color online) Decay exponents estimated from different quantities (case $\mathcal{B}$ : see Table I). Dashed line: $m_{\lambda}$. Dotted line: $m_{q}$. Dot-dashed line: $m_{\epsilon}$; Solid line: $t_{0}$. Vertical dashed lines indicate the time interval over which the averaged values of $t_{0}$ and $m$ are calculated.

compared to $m_{\epsilon}$ and $m_{q}$. These oscillations are not systematic but change from one simulation to another: a second simulation (not shown here) with the same initial power spectrum, but different random numbers defining the phase, gave similar mean values. Case $\mathcal{B}$ at higher $R_{\lambda}$ confirmed the accuracy of the method for evaluating $m$. Profiles of the instantaneous decay exponents are shown in Fig. 8, while the numerical values are listed in Table I. Compared to the lower$R_{\lambda}$ simulation, the initial decay is established at later times over the interval $10.1 \lesssim t \lesssim 11.8$, the origin $\left(\overline{t_{0}}=4.3\right)$ is larger, and the decay exponent $\left(\overline{m_{q}}=1.19\right)$ is smaller (see Table I). Similar oscillations in the values of $m_{\lambda}$ were also reported by Huang and Leonard [20] (their Fig. 8) for values of $R_{\lambda}$ close to the present ones. Further, as in our simulations, their distributions of $q^{2}$ seem to follow more closely a power law (their Fig. 2), in relation to those of $\lambda^{2}$.

The scatter in $m_{\lambda}$ can be shown to be inherent in the definition of $\lambda$. A small error $\delta_{\epsilon}$, in the estimate of $\epsilon$ from the power law of $q^{2}$, is reflected in a variation $\Delta$ of the decay exponent of $\epsilon-$ viz.,

$$
\delta_{\epsilon}=\frac{\epsilon^{*}}{\epsilon}=\frac{\left(t-t_{0}\right)^{-m_{\epsilon}+\Delta-1}}{\left(t-t_{0}\right)^{-m_{\epsilon}-1}}=\left(t-t_{0}\right)^{\Delta}
$$

(the asterisk denotes the estimate affected by the error), or $m_{\epsilon}^{*}=m_{q}-\Delta$. Consequently, the Taylor microscale is also affected by $\Delta$,

$$
\lambda^{* 2}=\frac{5 \nu q^{2}}{\epsilon^{*}}=\frac{10 \nu}{m_{q}}\left(t-t_{0}\right)^{1-\Delta} .
$$

Allowing for $\Delta$ does not mean that the energy equation (5) is incorrect, but rather that Eq. (6) is only a first-order approximation. The derivative of $\lambda^{* 2}$ is

$$
\frac{d \lambda^{* 2}}{d t}=\frac{10 \nu}{m_{q}}(1-\Delta)\left(t-t_{0}\right)^{-\Delta},
$$

which can be used to evaluate the effect of $\Delta$ on $m_{\lambda}^{*}$-i.e.,

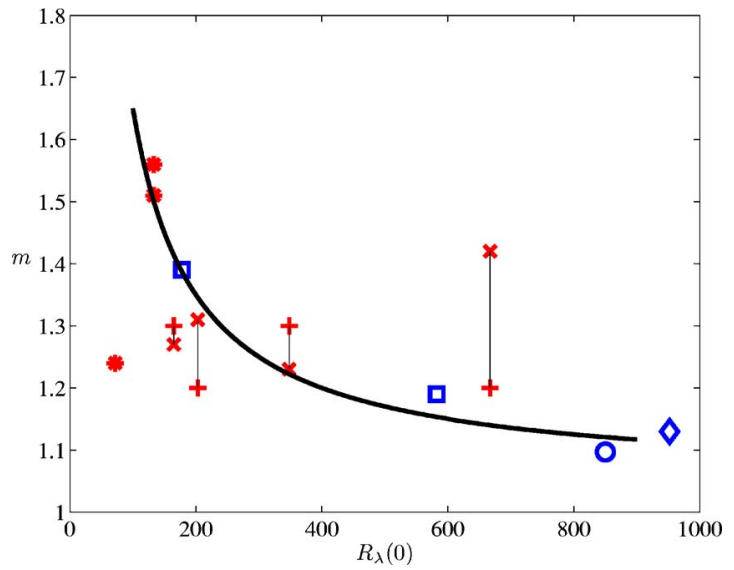

FIG. 9. (Color online) Dependence of the decay exponent (estimated from $\lambda^{2}$ ) on $R_{\lambda}(0)$, the Reynolds number at the beginning of the simulation. $(\square)$ present, $(\bigcirc)[8],(\diamond)[49]$, and $(+)[20] .(\times)$ data from [20] estimated via the decay of $q^{2}$. Vertical lines connect values of $m$ in [20] estimated from $q^{2}$ to those estimated from $\lambda^{2}$ [where the two estimates coincide, an asterisk $(*)$ appears instead]. The smooth thick line through the data, which is represented by $1.05+60 / R_{\lambda}(0)$, is drawn as a visual aid.

$$
\frac{m_{\lambda}^{*}}{m_{\lambda}}=\frac{d \lambda^{2}}{d t} / \frac{d \lambda^{* 2}}{d t}=\frac{\left(t-t_{0}\right)^{\Delta}}{(1-\Delta)} .
$$

For case $\mathcal{A}$, assuming $\delta_{\epsilon}$ is 1.1 (i.e., an error of $10 \%$ in the estimate of $\epsilon$ from the power law of $q^{2}$ ) in the initial period of decay $(t=5.5)$, Eq. (11) yields $\Delta=0.0641$. This is only an error of about $4.6 \%$ for $m_{\epsilon}$ but results in $m_{\lambda}^{*}$ being $12 \%$ larger than $m_{\lambda}$, according to Eq. (12). Thus, even a small uncertainty in $m_{\epsilon}^{*}$ has a large effect on $m_{\lambda}$. Indeed, Figs. 7 and 8 show that $m_{\lambda}$ has a larger variability, compared to $m_{\epsilon}$ and particularly $m_{q}$. The usual practice of determining $m$ via $q^{2}$ in experiments is hence justified, although it should be noted that, in doing so, an initial guess for $t_{0}$ is required. This can be obtained from Eq. (10), once an initial estimate for $m_{\lambda}$ is made.

Finally, we consider the dependence of $m$ on $R_{\lambda}(0)$, the value of the Reynolds number at $t=0$. Figure 9 shows our data, as well as data from other sources, and indicates that as $R_{\lambda}(0)$ increases, $m$ decreases. For some of the data in [20], there is a large difference between estimates based on $\lambda^{2}$ and $q^{2}$, thus raising some concern. This trend and the approach towards 1 for $m$ at large $R_{\lambda}(0)$ were also documented by George [18] for grid turbulence (in [18], the values of $m$ were plotted against $R_{\lambda}$ in the initial period of the decay), although earlier Mohamed and LaRue [17], who compiled values of $m$ from the literature, found that $R_{\lambda}$ had no systematic effect on $m$. Arguably, in box turbulence, initial conditions can be specified more precisely than in grid turbulence so that a possible dependence on $R_{\lambda}(0)$ or $R_{\lambda}$ can be discerned more easily.

\section{CONCLUSIONS}

Decaying homogeneous isotropic turbulence has been studied in a periodic box by solving the Boltzmann equation 
on a lattice. The results are found to be consistent with simulations made with different numerical schemes and with measurements of grid turbulence. In particular, the turbulent energy spectrum and the value of the velocity derivative skewness in the initial period of decay are very close to those measured in grid turbulence.

The power laws for the decay of the turbulent kinetic energy, the decay of the mean energy dissipation rate, and the growth rate of the Taylor microscale have been analysed. Estimates of the decay exponent $m$ obtained from these three quantities are very close, as required by theory. However, the power law for the turbulent kinetic energy is established first and displays smaller oscillations, compared to the mean energy dissipation rate and the Taylor microscale $\lambda$. The error propagation associated with the definition of $\lambda$ explains the larger uncertainty in $m_{\lambda}$. The present data, together with other values available in the literature, suggest that $m$ decreases towards 1 , as $R_{\lambda}(0)$ increases.

\section{ACKNOWLEDGMENT}

The support of the Australian Research Council is acknowledged.
[1] R. S. Rogallo (unpublished).

[2] D. Fukayama, T. Oyamada, T. Nakano, T. Gotoh, and K. Yamamoto, J. Phys. Soc. Jpn. 69, 701 (2000).

[3] R. A. Antonia and P. Burattini, J. Fluid Mech. 550, 175 (2006).

[4] S. M. de Bruyn Kops and J. J. Riley, Phys. Fluids 10, 2125 (1998).

[5] H. Wang and W. K. George, J. Fluid Mech. 459, 429 (2002).

[6] G. Comte-Bellot and S. Corrsin, J. Fluid Mech. 25, 657 (1966).

[7] P. Lavoie, P. Burattini, L. Djenidi, and R. A. Antonia, Exp. Fluids 39, 865 (2005).

[8] R. A. Antonia and P. Orlandi, J. Fluid Mech. 505, 123 (2004).

[9] N. N. Mansour and A. A. Wray, Phys. Fluids 6, 808 (1994).

[10] H. Yu, S. S. Girimaji, and L.-S. Luo, Phys. Rev. E 71, 016708 (2005).

[11] N. Satofuka and T. Nishioka, Comput. Mech. 23, 164 (1999).

[12] H. Yu, S. S. Girimaji, and L.-S. Luo, J. Comput. Phys. 209, 599 (2005).

[13] G. I. Taylor, Proc. R. Soc. London, Ser. A 151, 421 (1935).

[14] G. K. Batchelor and A. A. Townsend, Philos. Trans. R. Soc. London, Ser. A 193, 539 (1948).

[15] R. W. Stewart and A. A. Townsend, Philos. Trans. R. Soc. London, Ser. A 243359 (1951).

[16] G. K. Batchelor, The Theory of Homogeneous Turbulence (Cambridge University Press, Cambridge, England, 1953).

[17] M. S. Mohamed and J. C. LaRue, J. Fluid Mech. 219, 195 (1990).

[18] W. K. George, Phys. Fluids A 4, 1492 (1992).

[19] R. A. Antonia, R. J. Smalley, T. Zhou, F. Anselmet, and L. Danaila, J. Fluid Mech. 487, 245 (2003).

[20] M.-J. Huang and A. Leonard, Phys. Fluids 6, 3765 (1994).

[21] L. Djenidi, J. Fluid Mech. 552, 13 (2006).

[22] P. Bhatnagar, E. Gross, and M. Krook, Phys. Rev. 94, 511 (1954).

[23] Q. Zou and X. He, Phys. Fluids 9, 1591 (1997).

[24] R. S. Maier, R. S. Bernard, and D. W. Grunau, Phys. Fluids 8, 1788 (1996).

[25] P. Orlandi, Fluid Flow Phenomena. A Numerical Toolkit (Kluwer Academic, Dordrecht, 1999).
[26] S. B. Pope, Turbulent Flows (Cambridge University Press, Cambridge, England, 2000).

[27] G. Házi and C. Jiménez, Comput. Fluids 35, 280 (2005).

[28] P. Burattini and R. A. Antonia, Exp. Fluids 38, 80 (2005).

[29] P. Lavoie, Ph.D. thesis, University of Newcastle, 2006.

[30] T. Zhou and R. A. Antonia, J. Fluid Mech. 406, 81 (2000).

[31] L. Mydlarski and Z. Warhaft, J. Fluid Mech. 320, 331 (1996).

[32] H. S. Kang, S. Chester, and C. Meneveau, J. Fluid Mech. 480, 129 (2003).

[33] S. Tavoularis, J. C. Bennett, and S. Corrsin, J. Fluid Mech. 88, 63 (1978).

[34] J. R. Herring and R. M. Kerr, J. Fluid Mech. 118, 205 (1982).

[35] C. W. V. Atta and R. A. Antonia, Phys. Fluids 23, 252 (1980).

[36] T. Gotoh, D. Fukayama, and T. Nakano, Phys. Fluids 14, 1065 (2002).

[37] Y. Kaneda, T. Ishihara, M. Yokokawa, K. Itakura, and A. Uno, Phys. Fluids 15, L21 (2003).

[38] P. Burattini, P. Lavoie, and R. A. Antonia, Phys. Fluids 17, 098103 (2005)

[39] A. N. Kolmogorov, Dokl. Akad. Nauk SSSR 30, 299 (1941).

[40] K. R. Sreenivasan and R. A. Antonia, Annu. Rev. Fluid Mech. 29, 435 (1997).

[41] K. R. Sreenivasan, Phys. Fluids 10, 528 (1998).

[42] D. O. Martinez, S. Chen, G. D. Doolen, L.-P. Wang, and Y. Zhou, J. Plasma Phys. 57, 195 (1997).

[43] G. Comte-Bellot and S. Corrsin, J. Fluid Mech. 48, 273 (1971).

[44] W. K. George, H. Wang, C. Wollblad, and T. Johansson, in Proceedings of the Australasian Fluid Mechanics Conference, edited by B. B. Dally (Adelaide University, Adelaide, 2001), pp. $41-48$.

[45] J. Jiménez, A. A. Wray, P. G. Saffman, and R. S. Rogallo, J. Fluid Mech. 255, 65 (1993).

[46] L. P. Wang, S. Chen, J. G. Brasseur, and J. C. Wyngaard, J. Fluid Mech. 309, 113 (1996).

[47] P. K. Yeung and Y. Zhou, Phys. Rev. E 56, 1746 (1997).

[48] N. Cao, S. Chen, and G. D. Doolen, Phys. Fluids 11, 2235 (1999).

[49] A. Wray (unpublished). 